\title{
Uso de Tecnologias Computacionais para o Ensino de Crianças com Transtorno do Espectro Autista: um Mapeamento Sistemático da Literatura
}

\author{
Martony Demes da Silva ${ }^{1}$, Igo Coutinho Moura ${ }^{2}$, André Castelo Branco Soares ${ }^{1}$ \\ ${ }^{1}$ Universidade Federal do Piauí \\ Teresina - Piauí - Brasil \\ ${ }^{2}$ Instituto Federal do Maranhão \\ Timon - Maranhão - Brasil \\ mardemes@gmail.com, igocmoura@gmail.com, andre.soares@ufpi.edu.br
}

\begin{abstract}
Teaching autistic children demands the use of differentiated methods and tools, caused by cognitive, social and language difficulties of these individuals. Therefore, we have observed several published studies dealing with interventions that are supported by computational resources. This type of approach is effective in increasing the development of various skills, allowing for the advancement of the child's learning and enhancing the educator's work in his teaching practice. This work presents a systematic mapping of the literature with the purpose of investigating and interpreting the trends and gaps in the advances in the use of computational technologies for teaching children with ASD.
\end{abstract}

Resumo. Ensinar crianças autistas demanda o uso de métodos e ferramentas diferenciadas, ocasionado por dificuldades cognitivas, social e na linguagem desses indivíduos. Diante disso, observou-se diversas pesquisas publicadas tratando sobre intervenções que são apoiadas por recursos computacionais. Esse tipo de abordagem mostra-se eficaz no incremento do desenvolvimento de várias habilidades, possibilitando o avanço no aprendizado da criança $e$ potencializando o trabalho do educador em sua prática de ensino. Este trabalho apresenta um mapeamento sistemático da literatura com propósito de investigar e interpretar, as tendências e lacunas dos avanços no uso de tecnologias computacionais para ensino de crianças com TEA.

\section{Introdução}

O Transtorno do Espectro Autista (TEA) é um transtorno global do desenvolvimento caracterizado por gerar prejuízos nos indivíduos quanto às suas habilidades de interação social, comunicação, além da presença de comportamentos repetitivos e estereotipados [APA 2013].

Geralmente manifesta-se nos indivíduos ainda na infância. Nesta fase, a criança típica refina seu sistema neuromotor e desenvolve suas habilidades de comunicação, generalização, abstração e socialização.

Crianças com TEA enfrentam diversas dificuldades de aprendizado e 
alfabetização. Devido à existência de comportamentos atípicos que se manifestam de forma heterogênea e em diferentes níveis, realizar tratamento psicológico, fonoaudiológico, ocupacional, além do ensino e alfabetização, requer tempo e abordagens personalizadas que consideram o atual nível da criança e as suas demandas mais urgentes [Mattos and Nuernberg 2011].

Segundo o Center of Diseases Control and Prevention (CDC), órgão dos Estados Unidos equivalente ao Ministério da Saúde no Brasil, uma em cada 68 crianças são diagnosticadas com TEA naquele país [CDC 2015]. No Brasil, estima-se que existam mais de 2 milhões de indivíduos com TEA [OLIVEIRA, 2015]. Vale ressaltar que são dados preocupantes e que demandam mais atenção.

Recomenda-se, em crianças com TEA, realizar tratamento terapêutico com equipe multidisciplinar (psicologia, fonoaudiologia, psicopedagogia, terapia ocupacional, dentre outras.) [BRASIL, 2016]. O tratamento de uma criança com TEA demanda um esforço intenso, dedicação e acompanhamento do processo no consultório e domicílio. É comum que os resultados dados pela criança sejam a médio e longo prazo. O que pode gerar incertezas e frustrações na família. Em se tratando do ensino escolar, em especial na fase de alfabetização, os desafios tornam-se ainda maiores.

Crianças com TEA demonstram interesse em manusear e utilizar recursos computacionais (celular, tablet, jogos eletrônicos etc.). Por conta disso, recentemente, esses recursos são inseridos cada vez mais nas terapias e processos de ensino e aprendizagem dessas crianças [Passerino et. al. 2006].

Nesse sentido, o objetivo geral deste trabalho é identificar e investigar as tendências e lacunas dos avanços no uso de tecnologias computacionais para ensino de crianças com TEA, através de um Mapeamento Sistemático da Literatura, estudos primários sobre ferramentas computacionais que contribuem para o desenvolvimento do ensino de crianças autistas. Infere-se, a partir de pesquisas em bases de dados utilizadas neste trabalho, que não foram encontradas na literatura revisões formais com o mesmo objetivo.

No escopo deste trabalho considera-se como ferramentas computacionais os dispositivos móveis (tablets e smartphones), computadores de mesa (desktop) bem como dispositivos tecnológicos vestíveis como relógios, óculos, etc.

O restante deste artigo está organizado da seguinte forma: Na Seção 2 é descrita a metodologia de mapeamento sistemático utilizada no trabalho. A Seção 3 apresenta e discute os resultados obtidos no mapeamento. Por fim, a Seção 4 apresenta a conclusão.

\section{Metodologia do Mapeamento Sistemático}

Mapeamento Sistemático da Literatura é um método científico capaz de identificar, interpretar e sumarizar os trabalhos relevantes para determinada linha de pesquisa, área ou fenômeno de interesse de forma não tendenciosa e replicável [Kitchenham, 2007].

O MSL utilizado neste trabalho baseia-se nas diretrizes de trabalhos de [Kitchenham et al. 1995], que trata, de forma sistemática, os trabalhos desenvolvidos em uma determinada área do conhecimento e em seguida apresenta um resumo visual dos resultados encontrados. Para alcançar esses resultados, ele utiliza-se de um protocolo pré- 
VI Congresso Brasileiro de Informática na Educação (CBIE 2017)

Anais do XXVIII Simpósio Brasileiro de Informática na Educação (SBIE 2017)

definido. Nesse sentido, a condução deste trabalho subdividiu-se em três etapas: Planejamento, Execução e Sumarização/Resultados [Kitchenham, 2007].

\subsection{Planejamento}

Nesta etapa, o protocolo e a execução da pesquisa foram planejados. Preliminarmente os objetivos (citados na Seção anterior) foram modelados. Em seguida, as questões de pesquisa e as strings de buscas foram construídas. Logo depois, quatro bases de dados relevantes foram selecionadas nas quais aplicou-se as strings de busca. Ainda nesta mesma fase foram projetados os critérios de inclusão (CI) e os critérios de exclusão (CE) dos trabalhos obtidos. Por fim, os formulários de extração foram preparados e alinhados com os objetivos da pesquisa. Seguem os itens detalhadamente:

i) Questões de pesquisa: Para alcançar os objetivos citados, elencou-se questionamentos com uma Questão Principal (QP) e seis questões secundárias (QS). São elas:

QP: Como as ferramentas tecnológicas potencializam o desenvolvimento do ensino de crianças autistas?

QS1: Quais estratégias/abordagens da análise do comportamento para o ensino são utilizadas?

QS2: Quais as plataformas computacionais são utilizadas?

QS3: O sistema utilizado opera de forma centralizada ou distribuída?

QS4: Existem estratégias com uso de inteligência artificial?

QS5: A proposta computacional é adaptável às necessidades da criança?

QS6: Nas abordagens com uso de tecnologias, é comparado com o método tradicional?

ii) Strings de busca: Para responder às questões levantadas, inicialmente, foram construídas strings com palavras-chaves do tema da pesquisa. Propôs-se uma considerável quantidade de strings e seus sinônimos, com objetivo de chegar ao máximo de artigos relacionados ao assunto indagado. $O$ processo de busca foi realizado aplicando a string de pesquisa em diferentes bibliotecas adaptando ao mecanismo de busca de cada uma delas. As strings estão disponíveis em: https://goo.gl/MwexjG.

iii) Base de Dados: As strings foram submetidas às máquinas de buscas em quatro bases de pesquisas: Scopus, Web of Science, PubMed e APA. A escolha por essas bases de dados é justificada pelo objetivo de coletar o máximo possível de trabalhos com abrangência internacional. O período considerado na pesquisa foi de 2011 a 2016.

iv) Critérios de Inclusão (CI) e Exclusão (CE): Esses critérios são aplicados para selecionar os artigos a partir do resultado da coleta das bases de dados citadas. Considerando apenas os artigos primários, os trabalhos selecionados são apenas aqueles que estão de acordo com o critério de inclusão [Kitchenham, 2007]. Os critérios estão listados na Tabela 1 a seguir: 
VI Congresso Brasileiro de Informática na Educação (CBIE 2017)

Anais do XXVIII Simpósio Brasileiro de Informática na Educação (SBIE 2017)

Tabela 1. Critérios de Inclusão e de Exclusão

\begin{tabular}{|c|l|}
\hline & Descrição do critério \\
\hline CI-1 & $\begin{array}{l}\text { Trabalho aborda tecnologias computacionais que apoiem o ensino de criança no } \\
\text { espectro autista AND }\end{array}$ \\
\hline CI-2 & $\begin{array}{l}\text { Trabalho foi publicado e está disponível integralmente em base de dados científicas. } \\
\text { Idiomas considerados: inglês, espanhol e português }\end{array}$ \\
\hline CE-1 & Trabalho Não aborda tecnologias que apoiem o ensino a criança no espectro autista OR \\
\hline CE-2 & $\begin{array}{l}\text { Trabalho não disponível em formato digital ou impresso. Trabalhos que não seja nos } \\
\text { idiomas: inglês, espanhol e português. }\end{array}$ \\
\hline
\end{tabular}

v) Formulário de extração: Esse item é criado com base nas questões da pesquisa. É aplicado na etapa de execução, após a seleção, e objetiva encontrar os resultados quantitativos para as questões da pesquisa.

\subsection{Execução}

Nesta segunda etapa, foi posto em prática o que foi planejado na etapa anterior. Aplicouse as strings de busca nas quatro bases de dados. O resultado da busca foi um conjunto composto por 3.855 artigos pré-selecionados no formato bibtex. Logo em seguida, os arquivos nesse formato são inseridos em uma ferramenta de suporte a MSL a qual possibilitou realizar todas as etapas de maneira sistematizada. Mais informações sobre a ferramenta são descritas em [Braga, R. et. al. 2016]. O link da ferramenta está disponível em: https://goo.gl/yn7CBK.

Em seguida, dois pesquisadores (A e B) realizaram a seleção utilizando os critérios de inclusão e exclusão. Levou-se em consideração, inicialmente, a leitura do título e resumo dos trabalhos obtidos na busca. O resultado dessa seleção foi 218 artigos primários. Ressalta-se que essa quantidade inferior ao número inicial de artigos coletados nas bases de dados é coerente pois também ocorreu a remoção de artigos duplicados. Além disso, considerou-se apenas tecnologias computacionais especificadas na Seção 1.

Com objetivo de qualificar o trabalho, realizou-se uma análise de concordância entre os resultados obtidos pelos participantes. Para esta análise, os pesquisadores A e B realizaram um teste Kappa [Dixon and Massey, 1957]: Quanto maior o nível de concordância, maior a confiabilidade dos resultados. As divergências foram solucionadas por meio de discussão entre os pesquisadores. Dessa forma, dos 230 trabalhos que foram pré-selecionados, em 12 houve discordância quanto à seleção. Esses 12 trabalhos foram reavaliados por um terceiro pesquisador $\mathrm{C}$, que decidiu pela exclusão dos artigos, totalizando assim 218 aceitos.

Após a seleção, os artigos aceitos representam os trabalhos que responderão às questões de pesquisa definidas no protocolo do Mapeamento. Esses trabalhos foram lidos por completo pelos pesquisadores e em seguida os formulários de extração foram preenchidos. Diante desse contexto, obteve-se as informações relevantes para sumarização dos dados (próxima etapa). 
VI Congresso Brasileiro de Informática na Educação (CBIE 2017)

Anais do XXVIII Simpósio Brasileiro de Informática na Educação (SBIE 2017)

\subsection{Sumarização}

Nesta última etapa, os dados foram organizados em gráficos e tabelas. O objetivo dos dados é demonstrar quantitativamente um mapa das informações e visualizar as respostas para as questões da pesquisa. Para efeito estrutural, os resultados desse trabalho foram organizados na próxima Seção. A lista com os artigos aceitos e com detalhes dos dados sumarizados está disponível neste link: https://goo.gl/Nm2rHP

\section{Resultados}

Os resultados obtidos no MSL são apresentados e discutidos objetivando tratar as questões de pesquisa da metodologia aplicada. A Figura 1 apresenta a distribuição destes trabalhos pelas bases de dados escolhidas nos 6 anos.

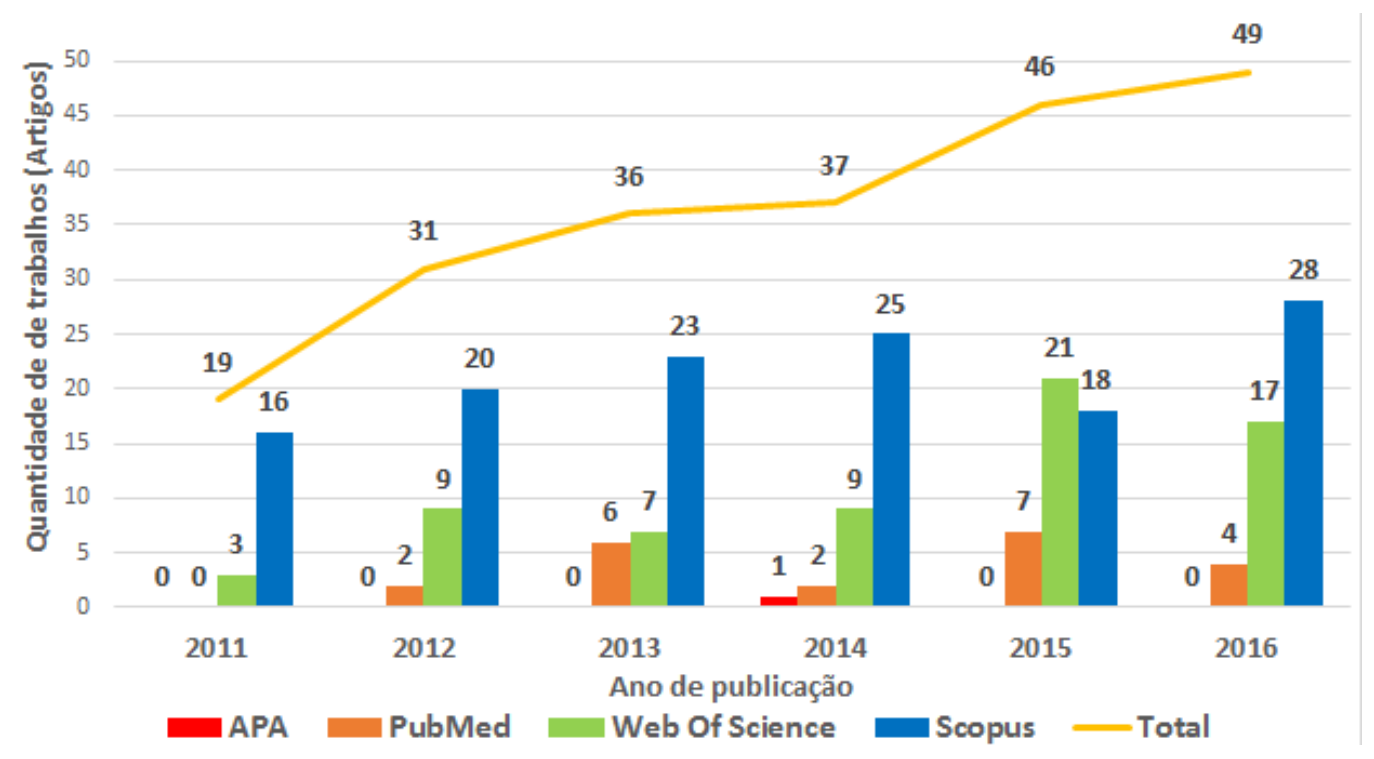

Figura 1: Distribuição de artigos por base de dados e ano de publicação

A linha contínua da Figura 4 apresenta o total de artigos publicados por ano. Como se observa, esse aumento significativo sugere, para esta linha de pesquisa, como um tema relevante. Assim, reforça o quanto pode ser interessante o uso de tecnologias para ensino de crianças com autismo. A seguir as respostas para as questões:

QS1: Quais estratégias/abordagens da análise do comportamento para o ensino são utilizadas?

Ao analisar o uso de abordagens (ou estratégias) que dão suporte ao ensino de crianças com TEA, pela Figura 2a, nota-se que há 185 (aproximadamente 84\%) que não utilizam uma estratégia de ensino. Presume-se uma lacuna para trabalhos futuros.

QS2: Quais plataformas computacionais são utilizadas?

A quantidade maior de plataformas utilizadas são tecnologias móveis (60\%), conforme mostra a Figura 2b. Com isso, infere-se que é tendência o uso dessas tecnologias que já são potencialmente utilizadas em intervenções no ensino de crianças com TEA. 
VI Congresso Brasileiro de Informática na Educação (CBIE 2017)

Anais do XXVIII Simpósio Brasileiro de Informática na Educação (SBIE 2017)

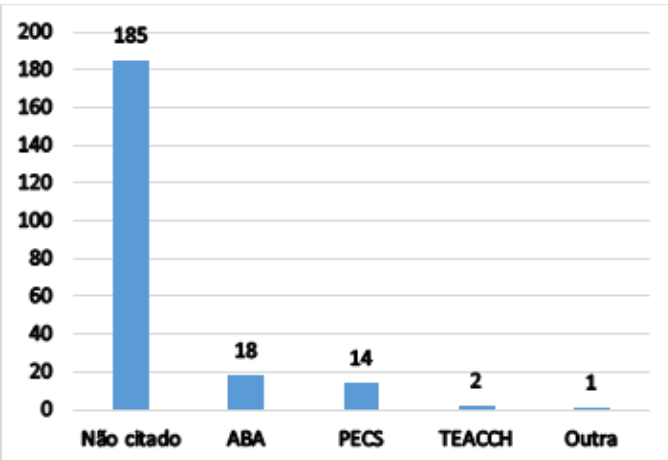

Figura 2 a): Número de abordagens/estratégias tratadas nos artigos

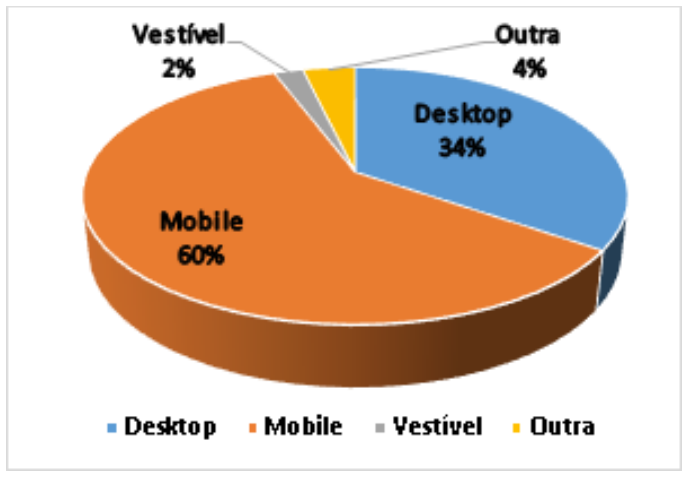

Figura 2 b): Quantitativo por plataformas computacionais

Outro destaque é a relação entre novas ferramentas propostas: para dispositivos móveis são 62\% (ou 90 novas ferramentas) e para desktop 61\% (ou 52 novas ferramentas), visto na Figura 3. Isso traduz que a maior parte dos trabalhos (tanto para mobile ou desktop) buscam propor novas ferramentas computacionais.

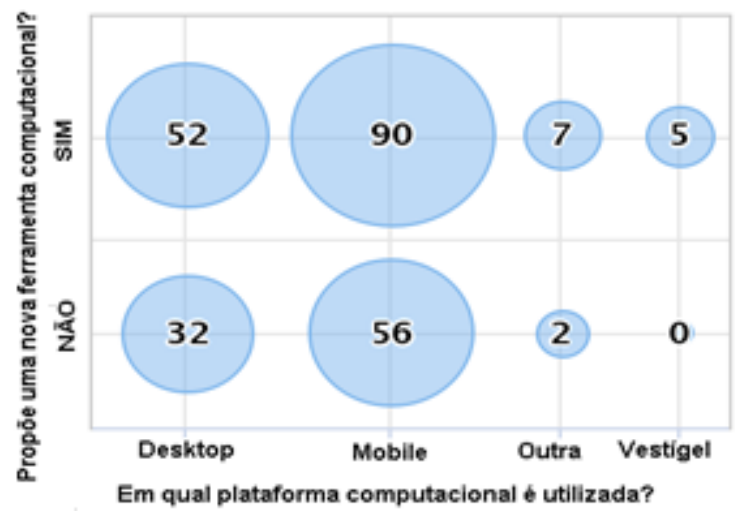

Figura 3: Quantitativo das plataformas computacionais utilizadas.

QS3: O sistema utilizado opera de forma centralizado ou distribuído?

O propósito deste questionamento é descobrir qual abordagem é mais utilizada nos trabalhos selecionados. Contextualiza-se, para este trabalho, que forma centralizada é os softwares interagem com uma base de dados centralizada. Partindo desse princípio, verificou-se, que há um quantidade razoável (47\%) dos trabalhos que operam de forma isolada. E o percentual é elevado, quando é comparado entre as plataformas computacionais. A Figura 4 ilustra que $65 \%$ das tecnologias móveis ainda trabalham de maneira isolada ou distribuída. 
VI Congresso Brasileiro de Informática na Educação (CBIE 2017)

Anais do XXVIII Simpósio Brasileiro de Informática na Educação (SBIE 2017)

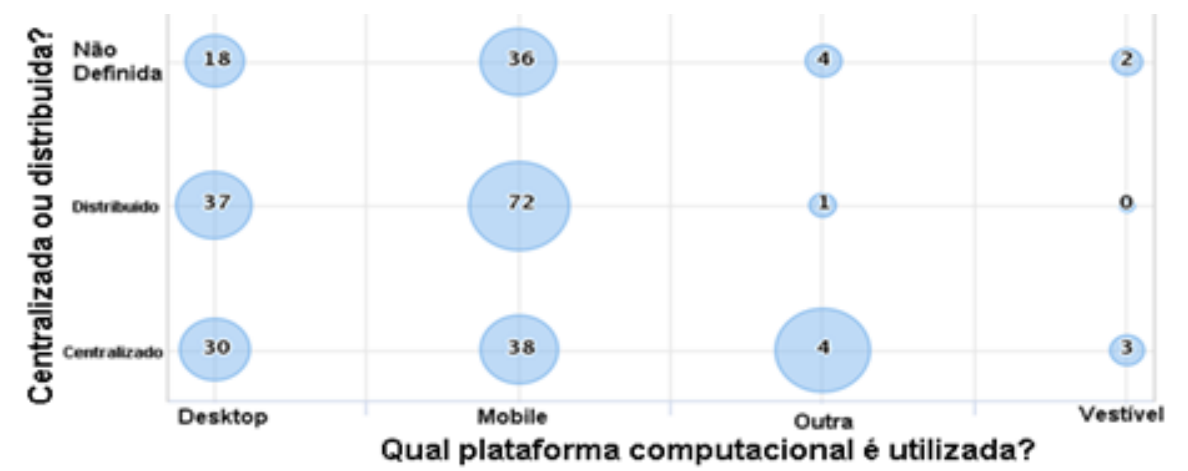

Figura 4: Comparativo entre abordagem distribuída/centralizada com plataforma tecnológica QS4: Existem estratégias com uso de inteligência artificial (IA)?

A utilização de Inteligência artificial (IA) ainda é pouco abordada nos trabalhos sobre tecnologias computacionais para ensino de crianças autistas- $6 \%$ ou 12 trabalhos apenas (Figura 5). Vislumbra-se, assim, oportunidades em pesquisa com uso de IA.

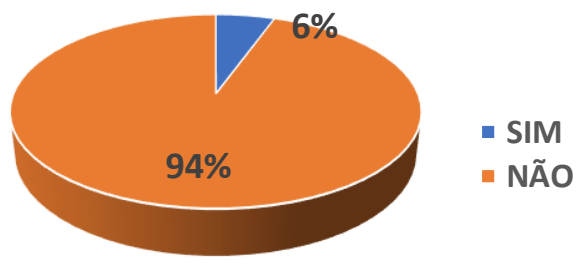

Figura 5: Estratégia utilizada faz uso de Inteligência Artificial?

Por outro lado, os trabalhos que propõem nova ferramenta computacional, apresentam um quantitativo relevante em utilizar IA (com 11 trabalhos) comparando-se estritamente com os trabalhos que não propuseram uma nova ferramenta computacional (apenas 1 com IA), ainda visualizado na Figura 6.

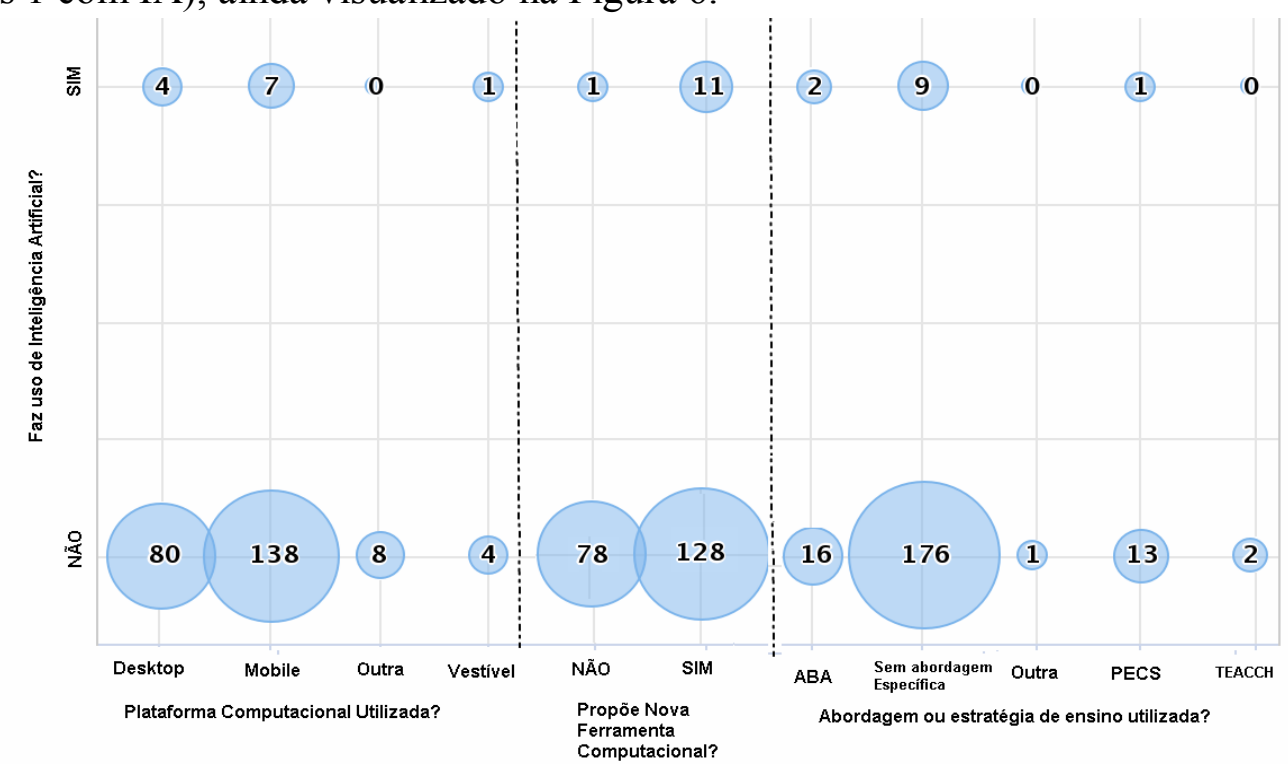

Figura 6: Comparativo com uso de Inteligência Artificial com: Plataforma Computacional, Nova Ferramenta e Abordagem de ensino. 
VI Congresso Brasileiro de Informática na Educação (CBIE 2017)

Anais do XXVIII Simpósio Brasileiro de Informática na Educação (SBIE 2017)

QS5: A proposta computacional é adaptável às necessidades da criança?

Por meio do gráfico gerado, disposto na Figura 7, tem-se uma perspectiva do quantitativo de trabalhos que apresentam ferramentas que permitem adaptação de atividades conforme o nível de desenvolvimento da criança. A maioria dos trabalhos $(87 \%)$ não permite personalizar as atividades com base no repertório da criança com autismo. Ressalta-se que em uma ferramenta computacional customizável, deve ser possível personalizar as atividades. Assim, permite-se construir atividades compatíveis com o desenvolvimento de cada indivíduo, evitando aquelas desnecessárias ou que são avançadas para seu estado atual.

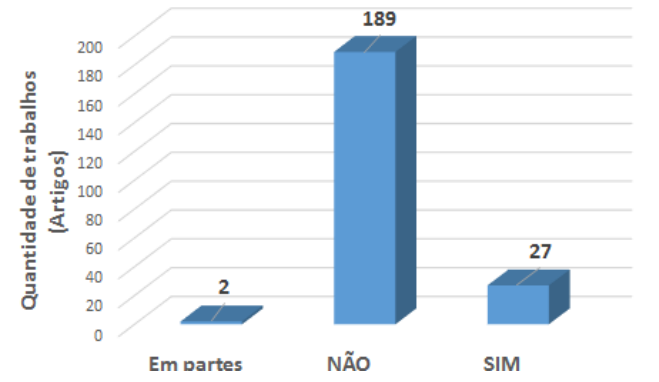

Figura 7: Número de artigos que apresentam ferramenta computacional adaptável às necessidades ou desenvolvimento da criança

A Figura 8 relaciona a possibilidade de adaptação da ferramenta de acordo com o nível da criança com outros três questionamentos?, são eles: i) É realizada a proposta de uma nova ferramenta computacional?, ii) A ferramenta alcança resultados melhores do que as abordagens tradicionais, iii) Qual a faixa etária considerada?

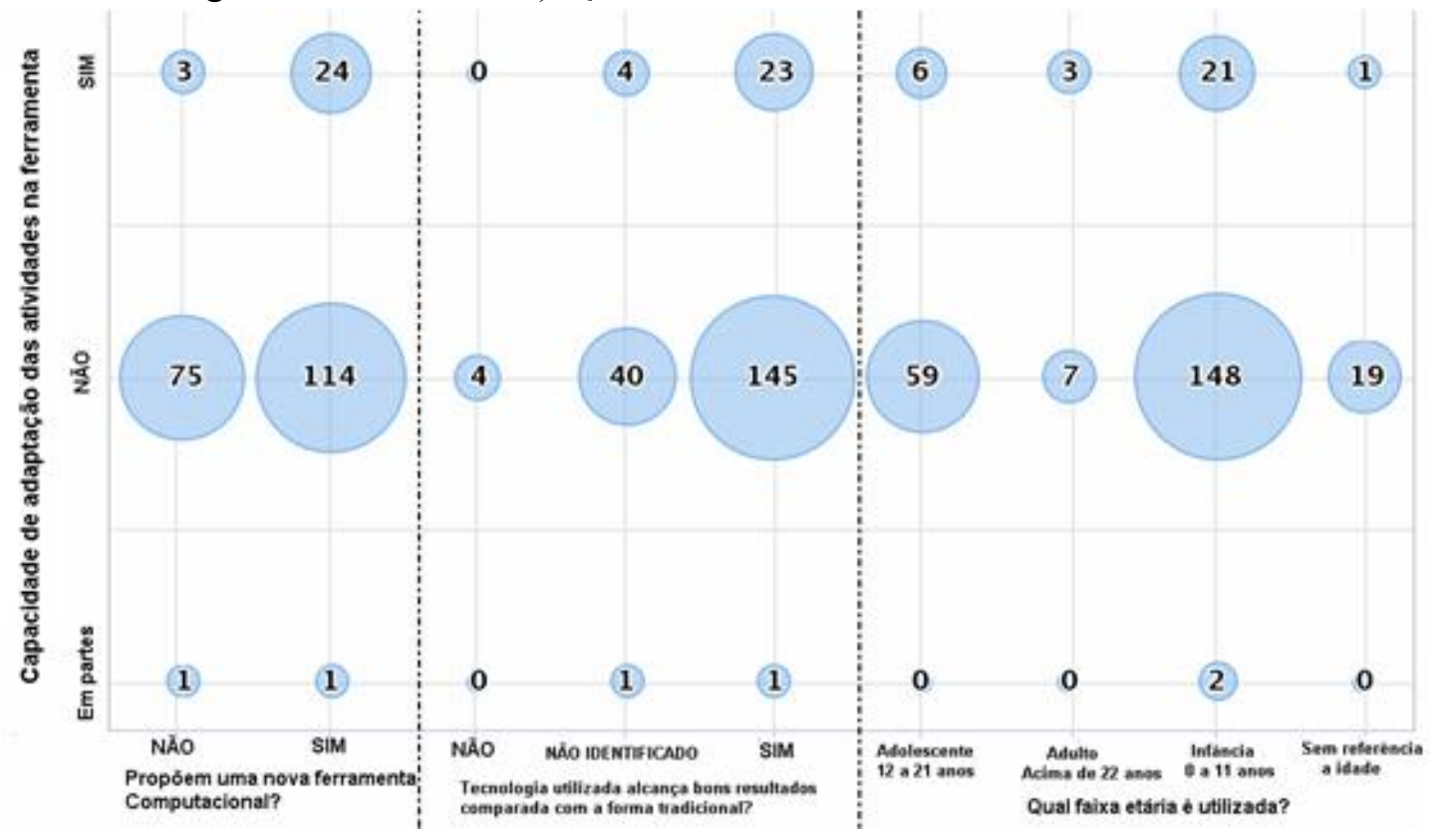

Figura 8: Número de artigos que apresentam ferramenta computacional de acordo com nível de desenvolvimento da criança relacionando a: nova ferramenta computacional, bons resultados comparado com forma tradicional e com a faixa etária. 
VI Congresso Brasileiro de Informática na Educação (CBIE 2017)

Anais do XXVIII Simpósio Brasileiro de Informática na Educação (SBIE 2017)

Diante dos valores apresentados dos trabalhos que permitem adaptação com nível da criança (27), visto na Figura 8, 24 trabalhos apresentam propostas de novas ferramentas computacionais. Ou seja, esses trabalhos que propõem ferramentas adaptáveis a maior parte são novas ferramentas propostas.

Outro destaque evidenciado: dos trabalhos realizados com adaptação ao nível de desenvolvimento da criança (27 trabalhos), ilustrado na Figura 8, 23 obtiveram resultados equivalentes ou superiores ao método tradicional (sem uso de tecnologias).

Conclui-se, como respostas à QS5, que há uma grande quantidade de tecnologias computacionais para faixa etária infantil ( 0 a 11 anos) com 171 trabalhos. Constata-se que há $67 \%$ dos trabalhos (ou 21 artigos dos 31) que também são direcionados para crianças na faixa etária até 11 anos. Isso nos remete que os trabalhos estão mais focados para crianças autista, que é o objetivo do ensino na idade certa.

QS6: Nas abordagens com uso de tecnologias, é comparado com o método tradicional? A resposta para esse quesito é sim. Ao examinar o gráfico da Figura 9a, a tecnologia utilizada alcança bons resultados (igual ou superior) - com 169 trabalhos ou $77 \%$ do total - quando comparada com a forma tradicional de ensino sem uso de tecnologia computacional. Esse argumento é reforçado, com a Figura 9b: dos 139 artigos que propõem uma nova ferramenta computacional, existem 120 que afirmam alcançar bons resultados se comparado com os métodos tradicionais não computacionais.

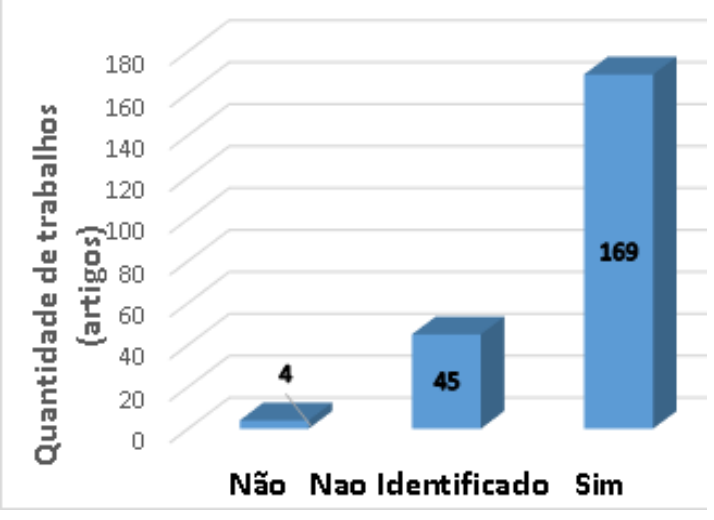

Figura ga: A Tecnologia alcança bons resultados comparados com método tradicional?

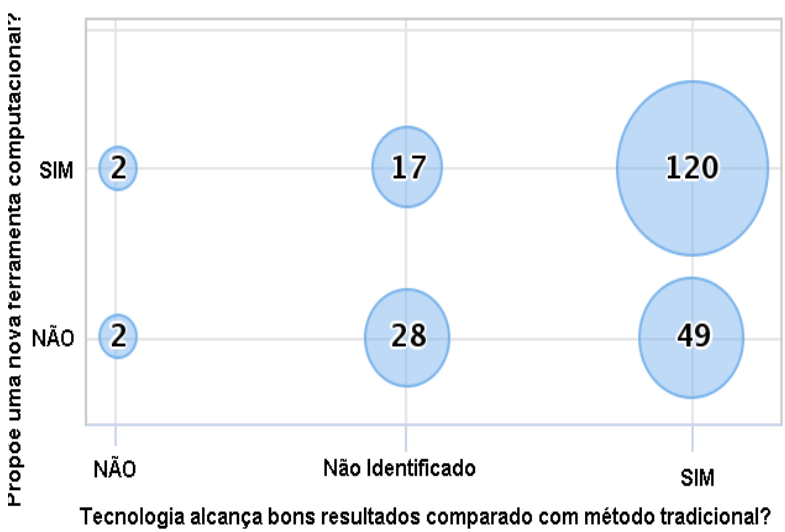

Figura gb: Número de artigos que apresentam nova ferramenta computacional relacionado com tecnologia alcança bons resultados comparado com método tradicional.

QP: Como as ferramentas tecnológicas potencializam o desenvolvimento do ensino de crianças autistas?

Para organização do trabalho, a resposta para este questionamento está descrita Seção 4 a seguir, conclusão deste trabalho. 
VI Congresso Brasileiro de Informática na Educação (CBIE 2017)

Anais do XXVIII Simpósio Brasileiro de Informática na Educação (SBIE 2017)

\section{Conclusão}

Conclui-se que foi necessário explorar todos os dados e gráficos expostos e levantar numericamente como as ferramentas possibilitam desenvolver o ensino de crianças autistas. Avaliando os resultados em todas as questões secundárias, as ferramentas tecnológicas expostas neste trabalho possibilitam o desenvolvimento do ensino de crianças com autismo. Ressalta-se, como forte fator de impacto, a evolução das tecnologias móveis. Outro elemento importante, dos trabalhos que propõem novas ferramentas, em sua maioria abordam sobre personalizar as atividades de acordo com nível de desenvolvimento da criança.

Destaca-se ainda algumas lacunas nas pesquisas, como moderados trabalhos com IA e necessidade de mais utilização de abordagens ou estratégia de ensino (como a ABA e TEACCH). Dessa forma, este trabalho contribui para o avanço na área da informática, fortalecendo o incentivo a futuros trabalhos na linha de tecnologias computacionais para desenvolvimento de crianças com autismo.

\section{Referência}

APA. American Psychiatric Association. Diagnostic and statistical manual of mental disorders. 5th ed. Arlington, VA: American Psychiatric Publishing, 2013.

CDC, Centers for Disease Control and Prevention. Estimated Prevalence of Autism and Other Developmental Disabilities Following Questionnaire Changes in the 2014 National Health Interview Survey. Disponível em: https://www.cdc.gov/ncbddd/autism/data.html. Acesso em 10 de maio de 2017.

BRASIL, Portaria No 324, de 31 de março de 2016. Aprova o Protocolo Clínico e Diretrizes Terapêuticas do Comportamento Agressivo com Transtorno do Espectro do Autismo. 2016. Disponível em: https://goo.gl/25pq9h. Acesso em 10 de maio de 2017.

Kitchenham, B., Charters S. Guidelines for performing Systematic Literature Reviews in Software Engineering. Vol 2.3 EBSE Technical Report, EBSE-2007-01, Software Engineering Group, School of Computer Science and Mathematics, Keele University, Keele, UK, (2007).

Dixon, W. J. and Massey Jr, F. J. (1957). Introduction to statistical analysis .

Braga, R., Oliveira, P.A., Souza, M., Andrade, G., Britto, R., Rabêlo, R. and Neto, P. S. Estudo Prático sobre Mapeamento Sistemático da Literatura utilizando a ferramenta TheEnd.Anais Escola Regional de Informática do Piauí, minicursos, p. 77-91, 2016.

Mattos, L. K. and Nuernberg, A H.. Reflexões sobre a inclusão escolar de uma criança com diagnóstico de autismo na educação infantil. Revista Educação Especial, n. 24 p.129-141. 2011.

OLIVEIRA, C. Um retrato do autismo no brasil. 2015.

Passerino, L.M., Santarosa, L.M C. and Tarouco, L. M. R. Pessoas com Autismo em Ambientes Digitais de Aprendizagem: estudo dos processos de Interação Social e Mediação. Anais do SBIE 2006. 